\title{
Biometric Features as a Guideline for the Best Colour Clothing Choice
}

\author{
Hadeel Farahat \\ October University of Modern Sciences and Arts \\ $6^{\text {th }}$ of October -Cairo-Egypt
}

\author{
Amr Elmagraby \\ October University of Modern Sciences and Arts \\ $6^{\text {th }}$ of October -Cairo-Egypt
}

\begin{abstract}
Nature is the most brilliant designer of all, and the secret is in her seasons. Each season presents a distinct array of colours, and your colouring is in harmony with one of these palettes. colour follows form and function, a common rationale and natural progression. your seasonal colour will give you the widest possible choice of shades that will harmonize with and enhance your natural colouring. The study will focus on uses computer for Biometry refers to a set of methods that rely on a set of distinctive, measurable characteristics in real-time human for skin colour detection and detection of eye colour and hair colour features.
\end{abstract}

\section{General Terms}

Pattern Recognition, Algorithms

\section{Keywords}

Seasonal colour; biometric features; cloth; algorithm

\section{INTRODUCTION}

To simplify the choosing of an individual's colour type, we take a cue from art educators, and every colour that exists is placed in one of four "seasonal" colour categories that match up with the colours of one of the four seasons of the year. Johannes Itten remarked that there wasn't a person in the world who couldn't successfully match up the four colour categories with the four seasonal categories.

The seasonal colour system is both logical and efficient: the two brightest seasons of the year, Spring and Summer, contain the brightest colours; and the two seasons with less daylight contain the colours that are the least bright, i.e., Autumn and Winter. [12]

Colour is reputed to be the first aspect of a product that consumers are drawn to and also for many designers it is one of the first of the fundamental elements of the design process to be considered. [11] In our colour analysis, we will use Munsell's Colour System.

The study will focus on the use of computers to determine to face biometric features and point to a set of methods that rely on a set of distinctive and measurable characteristics in humans in real-time to detect skin colour, discover eye colour, and hair colour features. Instead of using a tough, long-term workout with a coloured fabric under your face to see how your colouring (skin, hair, and eyes) interact to determine the proposed clothing colours that harmonize with our colouring to appear our faces and our personality.

After human face detection and determine facial parts (e.g. eye pupils, nose, lips, etc.) colour analysis feature we use Microsoft's newly released Face detection APIs to Guess Age and Gender-Based on a Photo We found the ability of the Face API to estimate age and gender to be particularly interesting and chose this aspect for our proposal. The proposed algorithm uses Microsoft Face API, to guesses a person's age and gender-based on a face to determine the proper cloth to fit.

\section{PREVIOUS RELATED WORK}

The current state of the art in the area is skin tone colour detection and skin colour analysis focus on the apparent skin colour is a result of different factors.

\subsection{Skin colour detection}

Skin tone in image detection is a very efficient way to locate skin-coloured pixels. Skin colour is a characteristic of human faces. In a controlled background environment, skin detection can be sufficient to locate faces in images. As colour processing is much faster than processing other facial features, it can be used as a preliminary process for other face detection techniques. [1]

\subsection{Skin Colour analysis}

1. Skin does not invert all light back at the surface light bounce inside and exit at different angles.

2. Blood acts like a numerous shading 'channels' engrossing and sifting light under the skin surface, making a lot of the exiting light red, resulting in a reddish hue.

3. Blood absorb and filter the light under the skin surface performing as a multiple colour 'filters', making a lot of the existing light red, resulting in a reddish hue.

4. Skin surface colour can change with sun exposure.

5. All drugs may induce skin reactions, skin surface colour can change with reactions to drug therapy and medication.

6. Veins determine skin colour cool-toned and warmtoned for example if your wrist veins appear bluer you are cool-toned and your wrist veins appear greener, you're warm-toned.

\section{DIMENSIONS OF COLOUR}

There are many obtainable Systems describing Colours. Munsell Colour System is one of the well-known systems, as it organises colour into clear order. Albert Munsell used three terms to describe the colour, as follow. [14]

HUE (Undertone - Warm or Cool)

Undertone is the fundamental quality of the colours, we refer the hue of colours, either yellow bases which are warm undertone or blue bases which is cool undertone and we have to know that any colour can have a cool or warm undertone.[4] 
VALUE (Depth - Light or Deep)

Describe the lightness and darkness for colours. There is a scale from 0 to 10.10 is white, 0 is the black and shades between 0 to 10 are greys.

\section{CHROMA (Clarity - Soft or Clear)}

Indicates the saturation or clarity of colour. Colours may be shining and clear, or muted and powdery. In the Munsell System, Chroma ranges from $0-14,14$ is the clearest and 0 is the most muted. [14]

\section{SEASONAL COLOUR ANALYSIS}

Each one of the four seasons has its main colour characteristics, which matches with people's personal colouring (skin, hair and eye) so we can categorize people into that four seasonal palettes. [7]

\section{Complexion}

Our skin has two main qualities:

\section{Undertone:}

which is the tone that underlying quality of the skin, determining whether your colouring is cool or warm. and it is a combination of 3 pigments:

- Haemoglobin - red

- Carotene - yellow

- Melanin - brownish-black

The amounts of haemoglobin and carotene indicate how much coolness and how much warmth are in the undertone. The amount of melanin indicates value and intensity.

So, there are three traditional undertones: warm, cool, and neutral.

1. Warm undertones span between peachy, yellow or golden tones.

2. Cool undertones contain traces of red, pink and bluish hues.

3.Neutral undertone is a blend of warm \& cool colours. It is roughly the same colour as your actual skin tone

Table 1. Different skin tones

\begin{tabular}{|l|l|}
\hline $\begin{array}{l}\text { warm } \\
\text { skin } \\
\text { tones }\end{array}$ & | \\
\hline $\begin{array}{l}\text { cool } \\
\text { skin } \\
\text { tones }\end{array}$ & \\
\hline $\begin{array}{l}\text { Neutral } \\
\text { skin } \\
\text { tones }\end{array}$ & \\
\hline
\end{tabular}

\section{Overtone}

Overtone is the obvious colour on the surface of our skin. but this colour can be affected by many factors like (Medications, disease, smoking, diet, pregnancy, etc..). [4]

Even though the overtone of the skin can change, the undertone will never change. The undertone can be specified despite any changing in the overtone.
Summers and Winters, are cool seasons, have blue undertones, while Autumns and Springs have golden undertones. [9]

$\underline{H A I R}$

Hair colour consists of around seven different tones of colours. During lifetime our hair colour changes, but it will keep in harmony with our eyes and skin. [4]

$\underline{E Y E S}$

The characteristic of the eye pattern and colours within it determine your season colour analysis, as any colour can be found in any season. [4]

\section{THE FOUR SEASONS 5.1 WINTER: cool, Deep, clear}

Winter people mostly have strong and obvious colours [6] and Winter people are the most common seasonal type all over the world [14]

\section{Skin:}

Winter people appear with rosy cheeks, although they have milky white skin and dark hair (like Snow White). The white may have an obvious pink tone or may appear pure white with a translucent quality. A great section of winters has taupebeige skin, ranging from light to dark, rosy or just plain beige. Most olive-skinned people, blacks, and Orientals are Winters, although a few are Autumns. [4] \& [9]

Hair:

Dark hair is the main colour for winters its rear to find an adult winter with the blonde tone, you can see them as whiteblonde only in their childhood. Brunette Winters hair colours ranged from light brown to dark charcoal brown, sometimes with a touch of red highlights. Some winter people have blueblack hair and silver grey. Of all the seasons, Winter is most likely to grey prematurely, but still looking attractive colour.
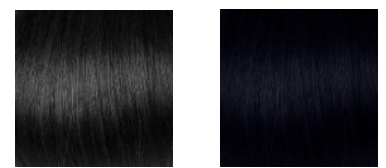

Eyes:

Winter's eyes are usually deep colours like red-brown, blackbrown, or hazel (a combination of grey-brown and blue or green); grey-blue, grey-green, or dark blue.

Most of the blue- or green-eyed Winters have white flecks in the iris. and some winters have a Grey-rimmed eyes.
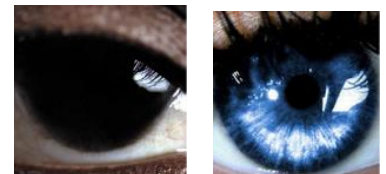

famous Winter figures: Elizabeth Taylor, Cher, Jacqueline Onassis, Audrey Hepburn, Jaclyn Smith, Marie Osmond, Princess Caroline Junot, Sally Field. [9]

\subsection{SUMMER: Cool, Muted, Light}

Summers are often blonde or wear blonde in their childhood then turned to light brown by days,

Skin:

usually have visible pink in their cheeks. Some of them have pink skin, with high colour, or rose-beige skin, ranging from fair to deep rose-beige. 
Hair:

Summers are almost blonde ranging between ash blonde to dark ash colour especially in their childhood, by growing up the hair tends to darken. in case of grey tone elegantly turn to a blue-grey or pearly white tone.

Brunette Summers have hair colour ranging from light to dark brown with ash overtones.
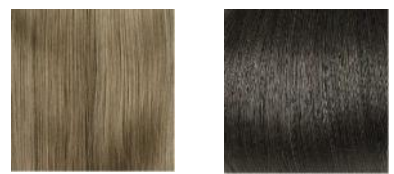

Eyes:

Most of the summers have Aqua, blue, green, or soft hazel (often green or blue with greyed brown framing the pupil.) few of them have soft brown eyes. Summer eyes are not dominantly clear or transparent, but cloudy and soft Grey summer people may also have eyes with a grey rim around the iris, and white flecks inside the iris.
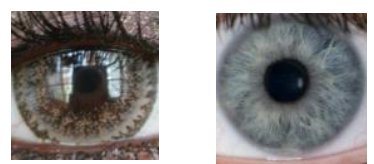

Famous Summer figures: Princess Grace of Monaco, Queen Elizabeth, Candice Bergen, Cheryl Tiegs, Farrah Fawcett, Nancy Kissinger, Caroline [9]

\subsection{AUTUMN: Deep, Warm, Muted}

Many Autumns are pale. Autumn and Springs may have similar colouring, but the Autumn generally with colourless cheeks, however, Springs have rosy cheeks. A few Orientals and blacks are Autumns if they have a truly golden undertone.

\section{Skin:}

Autumn people distributed in three types: a first type is a person with ivory or creamy peach skin they have a redhead, fair to dark, often with freckles; the second type is the goldenbeige brunette, who have copper tone ranging between medium to deep, and the last type found with charcoal black hair. Usually, you will find Reddish-brown freckles.[4]

\section{Hair:}

Autumn are usually with red-brown hair, sometimes they have a charcoal black hair several have golden blonde when they are young, going to darken by growing up. lots of Autumns are brunette, having gold or metallic red cast.
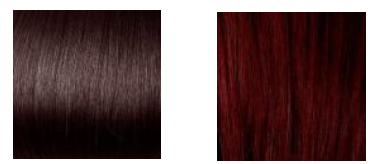

Eyes:

The generality of Autumns has brown eyes dark to topaz or green eyes predominantly sport gold or brown flecks in the iris. Hazel eye contains golden brown, green, and gold. Autumn eyes often with Isolated flecks, either gold, brown, or black. Clear green, olive green or vivid turquoise are few in Autumns.
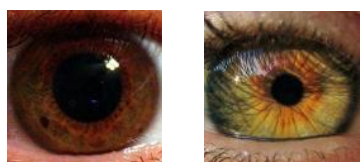

Famous Autumn figures: Vanessa Redgrave, Carol Burnett, Shirley MacLaine, Ann-Margret, Toni Tenille, Katharine
Hepburn, Lucille Ball, Maggie Smith. [9]

\subsection{SPRING: Light, Clear, Warm} Skin:

Most of the springs have finely textured skin which makes colours burst to the surface. they varied between the creamy ivory appears to have golden flecks, peachy pink and they are expected to have peachy-pink cheeks or peach-beige. Of all the seasons, Springs are the most likely to have rosy cheeks and to flush easily.

\section{Hair:}

Many Springs are blonde when they are young, but their hair going to darken when they grow up. The most common colours are ranging from golden blonde to strawberry, to taffy-red, or golden brown. Ash-tone hair is not Spring. Some have very dark brown hair. And a little of them have vivid carrot-tops.

Eyes
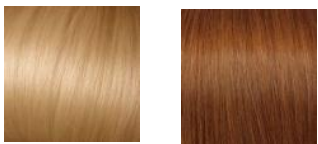

Blue, green, or aqua are the most dominant colours in springs, it could be clear like glass or with golden cluster surrounding the pupil. few springs have brown eyes but always they will be golden brown or topaz. A Spring's hazel eye contains golden brown, green, and gold.
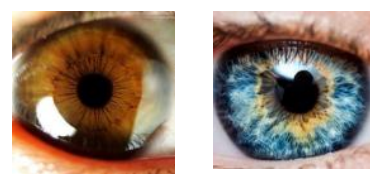

Famous Spring figures: Sally Struthers, Joan Kennedy, Zsa Zsa and Eva Gabor, Debbie Reynolds, Shirley Jones, Julie Andrews, Arlene Dahl, Marilyn Monroe. [9]

\section{THE FOUR SEASONAL PALETTES} WINTER

- Deep Winter is needed deep blue-based colours avoid light to mid-tones near the face.

- Cool Winter will be good in blue-based colours with medium Clarity and Depth.

- Clear Winter is good in high contrast blue-based colours, medium Depth.

\section{SUMMER}

- Cool Summer is good in different blue values from light to medium with low contrast and avoids yellow and gold.

- Muted Summer good in low contrast blue-based colours.

- Light Summer suits with very Light, blue-based, low contrast colours, Without Black.

\section{AUTUMN}

- Deep Autumn looks nice in two Deep, Warm minimal contrast colours.

- Warm Autumn fine with depth medium to deep all yellow-based colours with, low contrast.

- Muted Autumn matches very low contrast yellowbased colours, avoid black and hard white. (no contrast). 
Table 2. Seasonal colour palettes

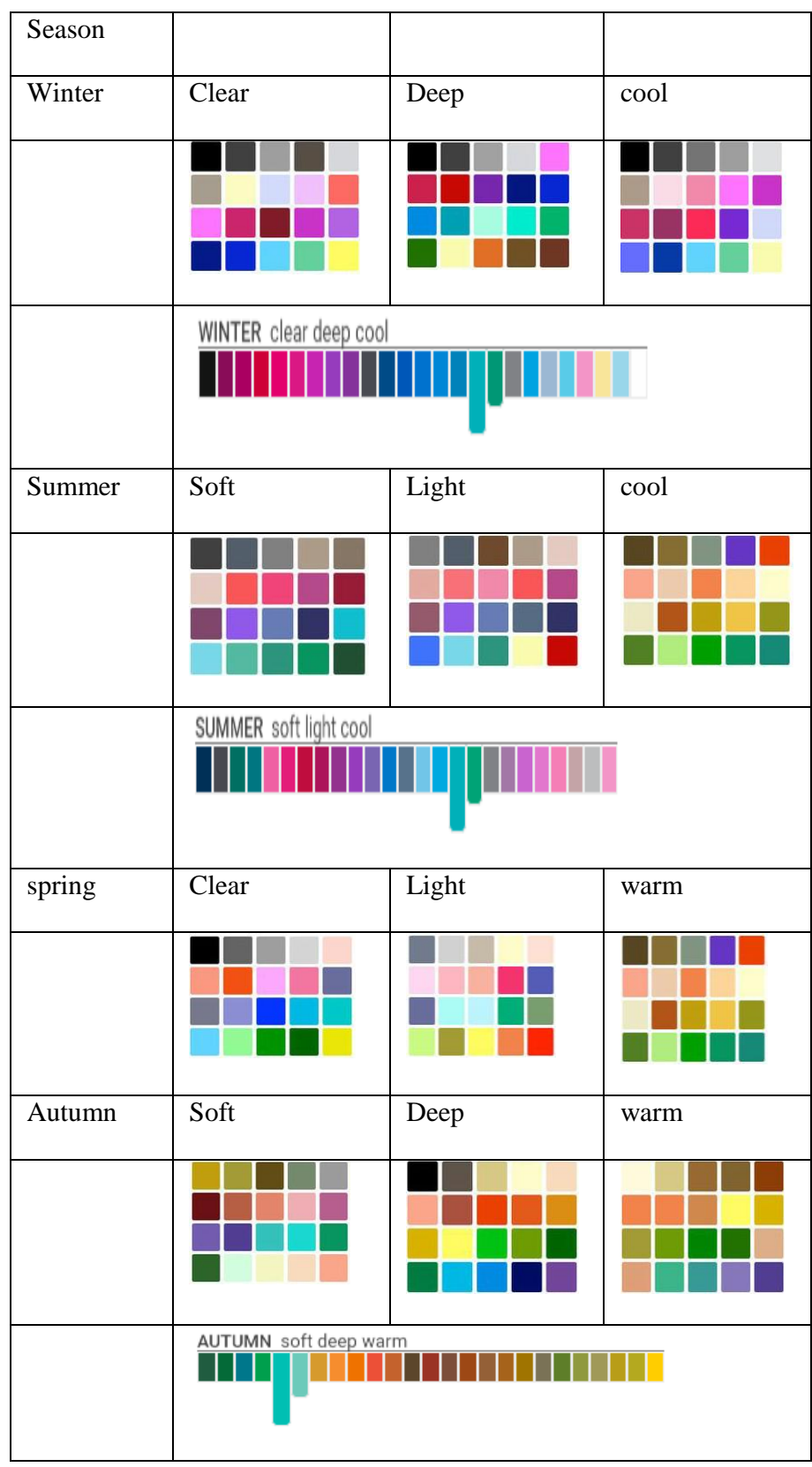

\section{$\underline{\operatorname{SPRING}}$}

- Light Spring matches the very Light, yellow-based, high contrast colours.

- Warm Spring; suits the yellow-based, medium to high contrast, medium to light colours.

- Clear Spring is good with high contrast yellowbased, medium to light colours.[5]\&[14]

\section{COLOUR PALETTES}

Deep

From its name this palette based on deep, strong and rich colours from the mid-tones to the darkest tone (almost black). this palette contains warm and cool colours, bright, clear and muted. Warm tones should be in contrast. The very light and icy tones could be used to achieve contrast or to add an accent. The colours of winter and Autumn are low in value, and neutral undertone - balanced between cool and warm. Light
In this palette, you can see colours between soft pastels and the mid-tones. Clear and bright or soft and muted.

In the light and mid-tones, colours will not look too bright, and you have to add light tones if using dark shades. The colours of summer and spring are high in value, and neutral undertone - balanced between cool and warm.

\section{Bright}

The Bright palette usually based on primary colours. ranging from mid-tones to deeper tones that remain clear and do not appear muted or greyed down.you have to use white or bright colours in case of using deep colours. As two deep colours together will appear heavy, as will muted shades. The colours of winter and spring are high intensity, and neutral undertone - balanced between cool and warm.

\section{Muted}

Muted colours can be warm or cool tones, they mainly are soft, blended, greyed-down colours range from mid-tones to rich deep colours. also, neutral colours and lighter shades considered as muted tones. The colours of summer and Autumn are low intensity, and neutral undertone - balanced between cool and warm.

\section{Warm}

The Warm palette colours with a clear golden undertone that range from mid-tones to deeper, rich tones, bright or muted. we can add greens and blues as an accent in the palette. The colours are between the spring and autumn palettes; warm, medium intensity, and medium value.

Cool

The Cool palette colours with blue undertones and ranged from mid-tones to deep shades. we have to add warm tones with the cool colours as an accent in the palette. The colours are between the winter and summer palettes; cool and medium intensity.[4]\&[13]

The simplest way to know your skin tone is comparing your skin colour with a white paper:

A. If the skin looks rosy pink or blueish it means you have cool skin tone.

B. If the skin looks greenish, yellowish or light brown it means you have warm skin tone.

C. If the skin looks ashen or greyish it means you have a neutral skin tone.

Table 3 Colours for different skin tones

\begin{tabular}{|l|l|l|l|l|}
\hline $\begin{array}{l}\text { Skin } \\
\text { tones }\end{array}$ & \multicolumn{4}{|l|}{ Colours for proper skin tones } \\
\hline Warm & \multicolumn{4}{|l|}{} \\
\hline $\begin{array}{l}\text { Colours } \\
\text { for warm } \\
\text { skin } \\
\text { tones }\end{array}$ & HONEY & OLIVE & CORAL & CREAM \\
\hline
\end{tabular}




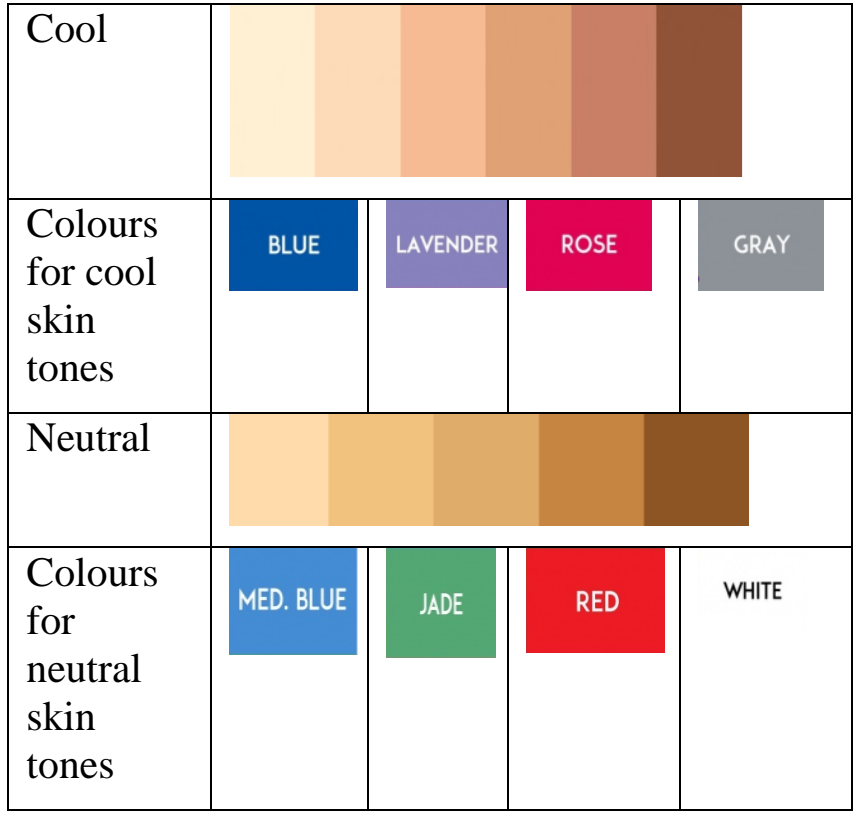

\section{METHODOLOGY}

The problem motivation in our research that Choosing clothing colours that flatter your skin tone only is a difficult task and don't give an accurate result. Our proposal considers skin tone as the main parameter with a Colour assessment like your hair, eyes, and skin will allow you to further define your category (warm or cool). Skin detection has also been used to locate body limbs, such as hands, as a part of hand segmentation and tracking systems, e.g., However, many objects in the real world have skin-tone colours, such as some kinds of leather, sand, wood, fur, etc., which can be mistakenly detected by a skin detector. Therefore, skin detection can be very useful in finding human faces and hands in controlled environments where the background is guaranteed not to contain skin-tone colours. Since skin detection depends on locating skin-coloured pixels, its use is limited to colour images, i.e., it is not useful with grey-scale, infrared, or other types of image modalities that do not contain colour information.

Several computer vision approaches have been developed for skin detection. A skin detector typically transforms a given pixel into an appropriate colour space and then uses a skin classifier to label the pixel whether it is a skin or a non-skin pixel. A skin classifier defines a decision boundary of the skin colour class in the colour space based on a training database of skin-coloured pixels. Different classes of colour spaces are the orthogonal colour spaces used in TV transmission. This includes YUV, YIQ, and YCbCr. YIQ which is used in NTSC TV broadcasting while $\mathrm{YCbCr}$ is used in JPEG image compression and MPEG video compression. One advantage of using these colour spaces is that most video media are already encoded using these colour spaces. Transforming from RGB into any of these spaces is a straight forward linear transformation.

The proposed framework is based on Transforming from RGB to HSV colour space and $\mathrm{YCbCr}$ chrominance space.[1].In this segment picture handling procedures and various tasks for various locales on a similar picture are applied as detail exploratory skin recognition.

\section{PROPOSED ALGORITHM}

The proposed method illustrated in this paper on the face detection task extends the cascade object detector framework proposed by Viola-Jones where objects are detected through four key concepts.

1. Simple rectangular features called Haar-like features.

2. Integral image for rapid features detection

3. AdaBoost machine-learning method

4. Cascade classifier to combine many features efficiently

Face part detection based on biometric features is not an easy problem since it requires multiple steps: a segmentation of a picture, identifying there is a person, then identifying a face on the person, segmenting hair and "skin" and finally detecting their colour. The accuracy percent depends on the image quality for our image procession. If we do not select a correct resolution, we may not get the desired result.[3]

Our proposed algorithm integrates all these object detector techniques by significant order to First detect the upper bodies of people using the 'Upper Body' classification model. Then detect the faces, and only keep the faces that are contained within upper bodies.

1- Upper Body Detector

2- Face detection (based on viola jones face detection or skin colour in the detection area).

3- Face part Classification is divided into six segments in which various parts of the face is detected.
a. Single Eye Detection.
b. Mouth Detection.
c. Eye Pair Detection.
d. Nose Detection.

Our experiment is done by Matlab's Computer Vision toolbox that provides vision.CascadeObjectDetector for object

detection, with support only for Frontal Face (CART),Frontal Face (LBP),Upper Body,Eye Pair,Single Eye,Single Eye (CART),Profile Face,Mouth,Nose.

The pose and position of the detected upper body are used for two purposes:

1- To estimate face region using viola jones face detection where the entire face must point towards the camera and should not be tilted to either side.

2- To estimate face region using skin colour detection in case of not get a result after Appling.

3- viola jones algorithm, to get fast and robust high detection rate. 
Table 4. Detect Upper Body in Image Using Upper Body Classification Model

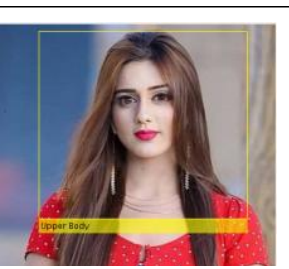

Create a body detector object and set properties.

bodyDetector $=$ vision. CascadeObjectDetector('UpperBody');

bodyDetector.MinSize = [60 60];

bodyDetector.MergeThreshold = 10;

Read input image and detect upper body.

I2 = imread('pic1.jpg');

bboxBody = bodyDetector(I2);

Annotate detected upper bodies.

IBody $=$

insertObjectAnnotation(I2,'rectangle',bboxBody,'Upper Body');

figure

imshow(IBody)

title('Detected upper bodies');

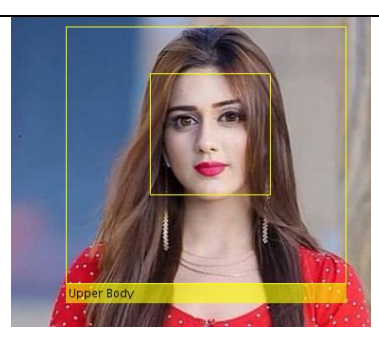

Face detection using Combination Haar-cascade training and skin colour form upper body.

I = imread('RIO.jpg');

Detect faces.

bboxes $=$ faceDetector(I);

Annotate detected faces.

IFaces $=$ insertObjectAnnotation(I,'rectangle',bboxes,'Face'); figure

imshow(IFaces)

title('Detected faces');

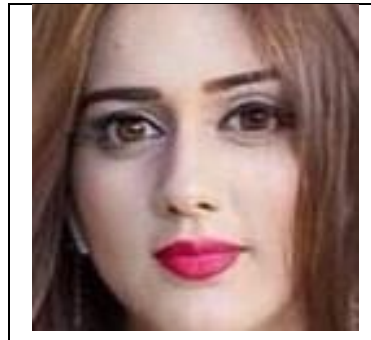

The location and colour information from the face can then be used to find limbs. As skin colour is a key visual feature in this system

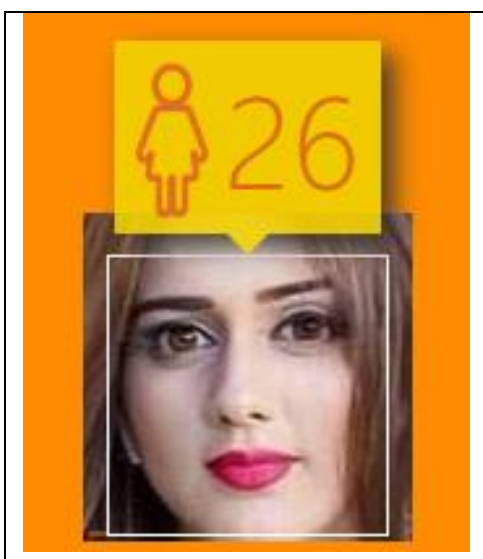

We use Microsoft internal API facial detection technology which uses facial-recognition technology to predict the age and sex of people.

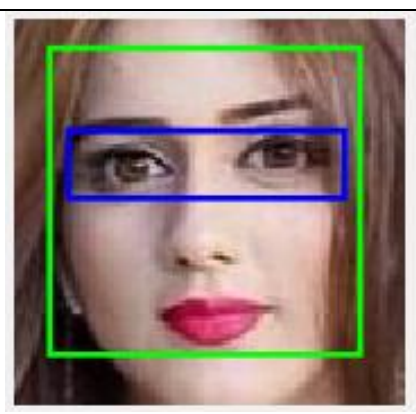

Face part detection : Eye Pair Big used to detect eye pair in the face detection area and create the region for performing the eyes search area of interest to detect eye.

EyeDetect $=$ vision .CascadeObjectDetector('EyePairBig');

BB_EyeDetect $=$ step $($ EyeDetect,I);

hold on

rectangle('Position',BB_EyeDetect,'LineWidth',2,'LineStyle','','EdgeColour','b');

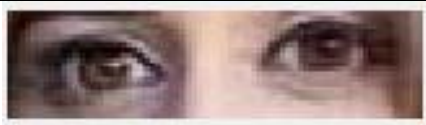

eye detection in a face image the detection rate was good ( $93 \%$ on the tested dataset) 


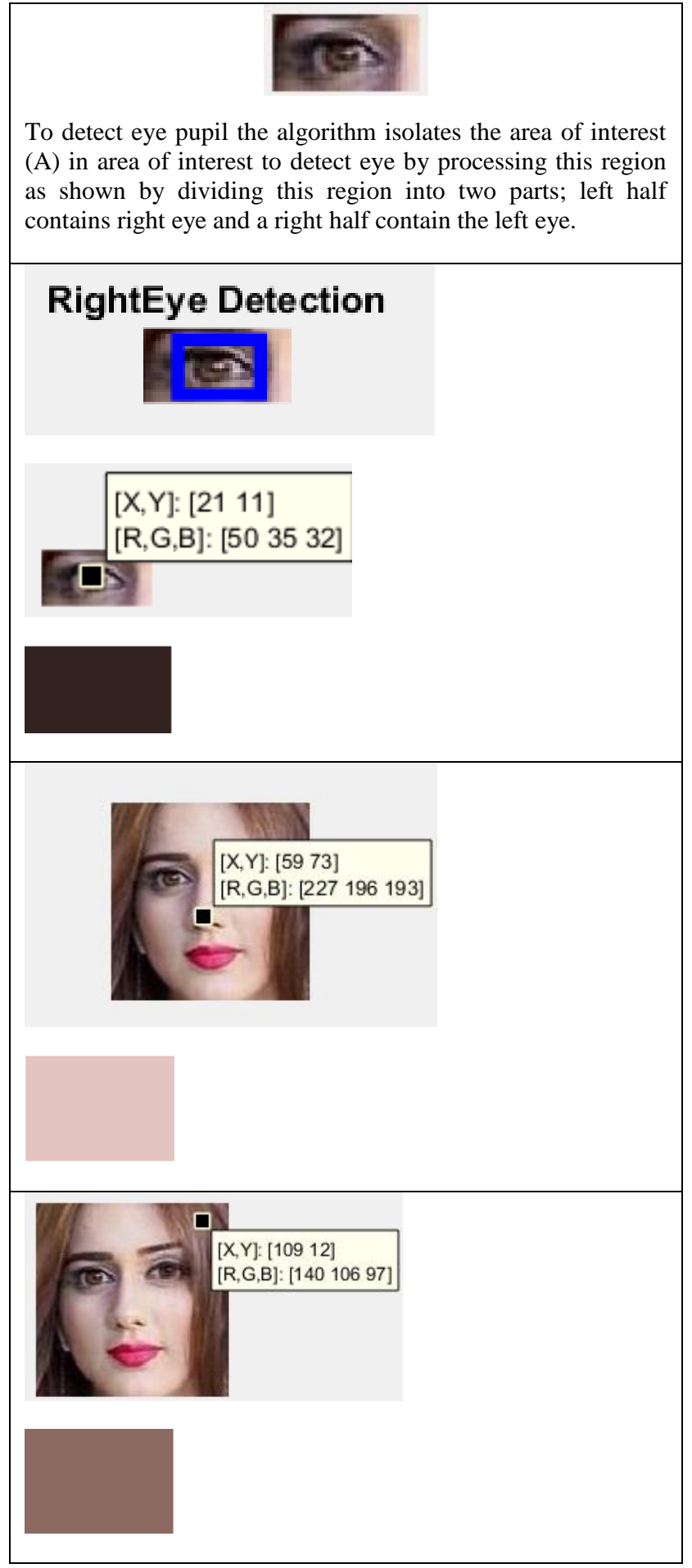

This is the primary detector where the high probability of finding the face instead of searching the entire image. To locate an exact face in that area of intrigue, Viola-Jones [3] face indicator is utilized as an auxiliary identifier to expand precision and decreases bogus negatives. Third indicator pixel-based skin recognition techniques are applied to the area of intrigue which isn't recognizing a face utilizing the auxiliary identifier. The third locator orders every pixel as skin or non-skin exclusively and autonomously from its neighbours and consolidates it with Viola-Jones chest area discovery. This improves the presentation of face identification frameworks regarding expanding the face discovery speed and diminishing bogus positive rate. The primary, secondary and third detectors are combined by this release and a single homogeneous set of face bounding-boxes are returned. The algorithm can detect a face in the video as usual in each image. Once a face is detected, a mixture of face detection and tracking from the point detected is used. The proposed algorithm able to obtains the unique functional features by enabling the proposed model to work with different skin colour tone, applying it to low-quality images, detecting faces with eyeglasses, determining the position of facial parts. [8]\&[10] The object detector utilizes the ViolaJones calculation to identify individuals' people's upper body. The model distinguishes the chest area district, which is characterized as the head and shoulders region. This model uses Hear features to encode the subtleties of the head and shoulder area. Since it utilizes more highlights around the head, this model is progressively powerful against present changes, for example, head revolutions/inclines To Detect Upper Body in an Image Using the Upper Body Classification Model. [3]

1- Create a detector object and set properties.

2- Read input image and detect upper body.

3- Annotate detected upper bodies.

The proposed algorithm locates the areas and sizes of human appearances in advanced pictures by extracting the region of interest and apply Viola and apply Viola-Jones legitimate article indicator in the region of interest for the request to restrict search territory. These methods drastically diminish the calculation time.

From the Detected area of eye pair, the region for performing the nose search is created as 1.5 height of eye region and the same width of eye pair (B) area of interest to detect nose. The region of the lower third of the face is isolated for performing the mouth search area of interest to detect mouth. To Detect the nose within the face region. The nose gives a progressively exact proportion of the skin tone since it doesn't contain any foundation pixels. Viola-Jones calculation is utilized as an initial step to Detect nose This model is made out of feeble classifiers, in light of a choice stump, which use Hair behaviour to encode nose details and drawing a bounding box relatively defined to the cropped face image. Adjusting the nose bounding box so that it is relative to the original image or video frame for detected supratip from the nose region. The model uses the following steps 1-the algorithm converts the nose detected colour image from BGR to HSV. 2-Split the HSV colour image into its separate Hue, Saturation and Value (Brightness). 3-Calculate maximum Brightness value returns the pixel of supratip.[1]\&[2]

\section{CONCLUSION}

The proposed study provides useful computer vision tools for colour analysis to help precisely determine your seasonal colour, giving you the widest possible selection of shades that blend with and enhance your natural colouration.

This proposed algorithm goes beyond the limits of all existing technologies as it obtains the unique functional features by enabling the proposed model to work with different skin colour tone, applying it to low-quality images, detecting faces with eyeglasses, determining the position of facial parts (e.g. eye pupils, nose, lips, etc.) and detect several faces in one image. As this software is typically designed to deal with single images. Results show that our algorithm improves performance by $98.98131 \%$ compared to the state of the art. 


\section{REFERENCES}

[1] Amr El Maghraby, N. A. E. Y. E., 2013. Hybrid Face Detection System using Combination of Viola - Jones Method and Skin Detection. International Journal of Computer Applications, Volume 71.

[2] Amr El Maghraby, N. A. E. Y. E., 2015. Detecting and Tracking of Multiple People in Video based on Hybrid Detection and Human Anatomy Body Proportion. International Journal of Computer Applications, Volume 109.

[3] Amr El Maghraby, N. M. A. E. Y. E., 2014. Detect and Analyze Face Parts Information using Viola- Jones and Geometric Approaches. International Journal of Computer Applications, Volume 101.

[4] Brunger, K., 2017. Colour Analysis. [Online] Available at: www.imageinstitute.com

[5] Cassidy, T. D., 2007. Personal Colour Analysis, Consumer Colour Preferences \& Colour Forecasting for the fashion \& Textile Industries. Colour design \& creativity, 6(1), pp. 1-14.

[6] Chiazzari, S., 1998. The Complete Book Of Colour. s.1.:Barnes \& Noble.
[7] D.Pooser, 1997. Always in style. Mississauge,Ontario: CrispLearning.

[8] Gomai, G., 2010. A new approach for pupil detection in iris recognition system (ICCET). Computer Engineering and Technology, Volume 4.

[9] Jackson, C., 2012. Colour me beautiful. Washington : Acropolis books Ltd.

[10] M. Hassaballah, K. a. S. I., June 13-15, 2011, . Eye and Nose Fields Detection From Gray Scale Facial Images. Nara, JAPAN, s.n.

[11] McKelvey, K. \&. M. J., 2011. Fashion design: process, innovation and practice. s.1.:John Wiley \& Sons.

[12] Nasr, M., 2018. Identifying the role of skin tone in choosing the suitable colors for outfits \& fashion accessories. International Journal of Textile and Fashion Technology (IJTFT), 1-14 June.8(3).

[13] Pooser, D. S. M. A. A., 2010. Always in Style. second edition, ed. USA: EBSCO Publishing.

[14] Wilkinson, J., 2020. [Online] Available at: http://colourmebeautiful.co.nz/wpcontent/uploads/2013/11/colour-training-on-line.pdf 